\title{
El comportamiento de la audiencia lineal, social y en diferido de las series de ficción españolas
}

The behaviour of the linear, social and time-shifted audience of Spanish fiction series

Ana González Neira, Universidade da Coruña - agonzalezn@udc.es

Natalia Quintas Froufe, Universidade da Coruña - n.quintas.froufe@udc.es

\section{Resumen}

La introducción de nuevas formas de consumo televisivo lleva consigo cambios en las audiencias. En un contexto de televisión líquida se hace cada vez más necesario analizar nuevos tipos de consumo como el que se realiza en las redes sociales (audiencia social) 0 el visionado de productos televisivos fuera del horario de programación (audiencia en diferido). En este artículo, a través del estudio de caso, se analizan tres tipos de audiencia (lineal, social y en diferido) de tres productos de ficción emitidos en las televisiones generalistas españolas durante el último trimestre de 2015. De la investigación se concluye que el éxito en la audiencia lineal no asegura la superioridad en la audiencia social, si bien los datos de las tres se complementan y nos acerca al nuevo televidente.

\section{Palabras clave}

Televisión, televisión social, redes sociales, audiencia social, audiencia lineal, audiencia en diferido, ficción.

\section{Abstract}

The introduction of new forms of television consumption leads to changes in the audiences. In a context of liquid television it becomes increasingly necessary to analyze new types of consumption as it is done on social media (social audience) or viewing television products after hours of programming (time shifted). This article analyzes three types of audience (linear, social and time shifted) of three fictions cast in the Spanish mainstream television during the last quarter of 2015. The investigation concluded that success in linear audience does not ensure superiority of the social audience, although the data of three complement each other.

Keywords

Television, social television, social media, social audience, linear audience, time shifted audience, fiction.

Sumario

1. Introducción. 2. Metodología. 3. Resultados. 4. Conclusiones. 5. Referencias bibliográficas. 


\section{Introducción}

Los recientes cambios en las formas de visionado de la televisión han alterado profundamente el panorama de las audiencias de este medio. En apenas unos años se ha producido una hibridación de la televisión al entrelazarse con dispositivos móviles, redes sociales y plataformas como Netflix o Youtube. La mejora en la calidad y velocidad del acceso a internet sin duda ha facilitado estas transformaciones.

De este modo, cada vez resulta más complejo definir qué es ver la televisión ya que estamos lejos de esas escenas en las que en el salón de un hogar la familia se reunía en torno al televisor durante la emisión de un programa concreto (Abreu et al., 2016). Ante aquellos que apuntan la muerte de la televisión, cabe indicar que esta se ha beneficiado y enriquecido con la aparición de estas nuevas especies en el ecosistema mediático. Estamos ante unas audiencias interactivas, conectadas a través de las redes sociales, que seleccionan sus contenidos más allá de las parrillas diarias, «consumatori selettivi e orientati alla ricerca di contenuti da trasformare in eventi mediali» (Marinelli \& Andò, 2016: 7).

En estos momentos, la multiplicidad de pantallas, el acceso online y los nuevos sistemas de grabación están alterando la forma de consumir estos contenidos televisivos dando mayor peso al espectador que decide cómo, cuándo y dónde verlos. Los avances técnicos le han brindado mayores posibilidades para interactuar con el tradicional emisor. Existe por lo tanto una tendencia a la personalización de contenidos, inexistente hace décadas. Las audiencias activas se dotan progresivamente de mayor poder y rompen los esquemas tradicionales de la audimetría.

Como indican Marinelli y Andó (2016), esta nueva realidad es posible también gracias la circulación de contenidos, que ya no están limitados a las parrillas fijas, sino que son accesibles desde otras plataformas como las aplicaciones o la web. Se sustituye el flujo unidireccional de los contenidos por una corriente circular y continua, que facilita la personalización del consumo por parte de la audiencia. Se trata de un elemento más de lo que se conoce como televisión líquida, en la que los tradicionales flujos se han visto radicalmente alterados (González-Neira, A. \& Quintas-Froufe, 2015: 143). Es también la televisión, como indica Monzoncillo (2011), del anyone, anywhere, anytime que lleva consigo una nueva dictadura del espectador, con contenidos cross media y con conexión permanente (always on), concepto que también recoge Díaz Nosty (2015).

A diferencia de lo que sucedía hace unos años, los contenidos están disponibles desde diferentes dispositivos y siempre, por lo que actividad de la audiencia se alarga y rompe los tiempos cerrados de la tradicional parrilla televisiva. En la época de esplendor de las series ha surgido con fuerza el binge watching tan típico del fenómeno fandom. Esta ruptura temporal se produce no solo en lo que respecta al visionado, sino también a los comentarios o reacciones en redes sociales (López-Meri, 2015: 35), que a su vez prolongan la vida de contenido televisivo visto más allá de su inicial emisión. Además cada vez más frecuentemente se están construyendo estrategias transmedia que avanzan en esta dirección como lo han demostrado Barra y Scaglioni (2014: 111).

La necesidad de conocer a estos consumidores, tanto para los directivos de las cadenas tradicionales como para el mercado publicitario, impulsa la puesta en marcha de nuevas técnicas que brinden mayor información (Cascajosa, 2016: 58) y se encaminen al ATAWAD (any time, any where, any device). Si bien los tradicionales audímetros siguen ofreciendo datos valiosos sobre el consumo tradicional o lineal, es necesario poner en marcha otras estrategias de recogida de información que hagan frente a estas nuevas audiencias (Kosterich \& Napoli, 2016). La publicidad necesita conocer quién está detrás de los comentarios de un programa en Twitter o quién accede a un contenido durante los días posteriores a la emisión para afinar más sus mensajes.

Estos recientes retos que suponen grandes cambios en el panorama televisivo han sido abordados por investigadores como Andò (2014), Portilla (2015), Abreu et al. (2016), Carey (2016) o Cascajosa (2016), entre otros. A los estudios tradicionales de audiencia, se han sumado en los últimos años algunas investigaciones acerca de la audiencia social. En este sentido debemos destacar las aportaciones sobre comparativas entre la audiencia social y aquella tradicional en España de Gallardo-Camacho, Lavín y Fernández-García (2016a y 2016b) y González-Neira, \& Quintas-Foufe (2014); aquellas de Saavedra, Rodríguez y Barón (2015), Deltell y Claes (2015), Deltell, Claes y Congosto (2015) sobre la ceremonia de los Premios Goya o de Iñesta Fernández (2015), Gómez Rubio y López Vidales (2015) 0 Alonso (2014) acerca del nuevo papel del espectador y su participación. En el panorama internacional investigadores como Kosterich (2016); Suing, Gallardo y Ortiz (2016); Steele et al. (2015); Woodford, Goldsmith y Bruns (2016); Simons (2015); Min, Zang y Liu (2015) o Bredl et al. (2013) han abordado la aparición de estas nuevas audiencias. No obstante, al tratarse de realidades tan recientes, y en parte poco estandarizadas como la audiencia en diferido, esta casi no ha sido objeto de análisis exceptuando a Evens y Berte (2014), el ya mencionado de Portilla (2015) o los recientes de Abreu et al. (2016) y Cascajosa (2016).

\section{Metodología}

La finalidad de esta investigación es profundizar en el conocimiento y comportamiento de los tres tipos de audiencia (lineal, social y en diferido) que puede tener un producto audiovisual, en este caso concreto, una serie de ficción nacional emitida en una cadena generalista española. Se ha optado por las series de ficción ya que en el año 2015 el 41,9\% del tiempo de emisión en televisión se destinó a la ficción y los espectadores españoles dedicaron más del 40,6\% de su tiempo a su visionado (Zenith, 2016).

Los objetivos de investigación son: en primer lugar, conocer cuál es el comportamiento de cada una de las audiencias (lineal, en diferido y social) en relación a tres series de ficción españolas y en segundo lugar, tratar de establecer un análisis comparativo de las mismas.

Se parte de la premisa de que la audiencia en diferido puede lograr incrementar considerablemente la audiencia de un producto de ficción, llegando a concentrar un mayor número de espectadores que el visionado lineal. Como señalaron Madinaveitia y Merchante (2015: 30) en determinadas series (sobre todo las de producción norteamericana) la audiencia en diferido puede triplicar a la audiencia en vivo. 
La metodología seleccionada para esta investigación fue la del estudio de caso (Yin, 1989) al ser la más adecuada para mostrar un análisis en profundidad de esta nueva realidad. Este caso, de carácter exploratorio y analítico según Coller (2000), pretende indagar sobre las diferentes formas de visionado de un producto audiovisual que se miden en la actualidad en el ámbito español.

El indicador utilizado para la selección de los canales fue la cuota de pantalla anual. Se seleccionaron las cadenas de televisión en abierto de carácter generalista que contaran con una mayor cuota de pantalla en el año 2015. Estos canales fueron Telecinco (14,8\%), Antena 3 $(13,4 \%)$ y La $1(9,8 \%)$. Posteriormente se escogió una serie de ficción emitida en prime time durante el inicio de la temporada televisiva 2015-2016 (mes de septiembre) de cada una de las cadenas. El periodo de análisis abarcó desde septiembre a diciembre de 2015.

Los productos de ficción seleccionados fueron los siguientes:

a) $B \& B$ (de Boca en Boca) (trece capítulos de la segunda y última temporada) producida por Globomedia y Mediaset España y emitida por Telecinco desde el 16 de septiembre de 2015, recrea la redacción de una revista de moda en España.

b) Velvet (quince capítulos de la tercera temporada) producida por Bambú Producciones y emitida por Antena 3 desde el 10 de septiembre de 2015, está ambientada en los años sesenta en las galerías comerciales Velvet.

c) Carlos Rey Emperador (doce capítulos de la primera y última temporada) producida por Diagonal TV y RTVE y emitida por La 1 desde el 7 de septiembre de 2015, narra la vida del emperador Carlos V.

Los principales indicadores seleccionados para el análisis de las tres categorías de audiencia fueron: en relación a la audiencia lineal: el número de espectadores y la cuota de pantalla; respecto a la audiencia en diferido: el número de espectadores VOSDAL + ADE (1-7) y para la audiencia social, la audiencia única total en Twitter.

Los datos manejados en este artículo fueron facilitados directamente por Kantar Media.

\section{Resultados}

A continuación se muestran los datos de los tres tipos de audiencia objeto de estudio en este artículo.

Gráfico n¹. Número de espectadores (expresados en miles) de las series de ficción

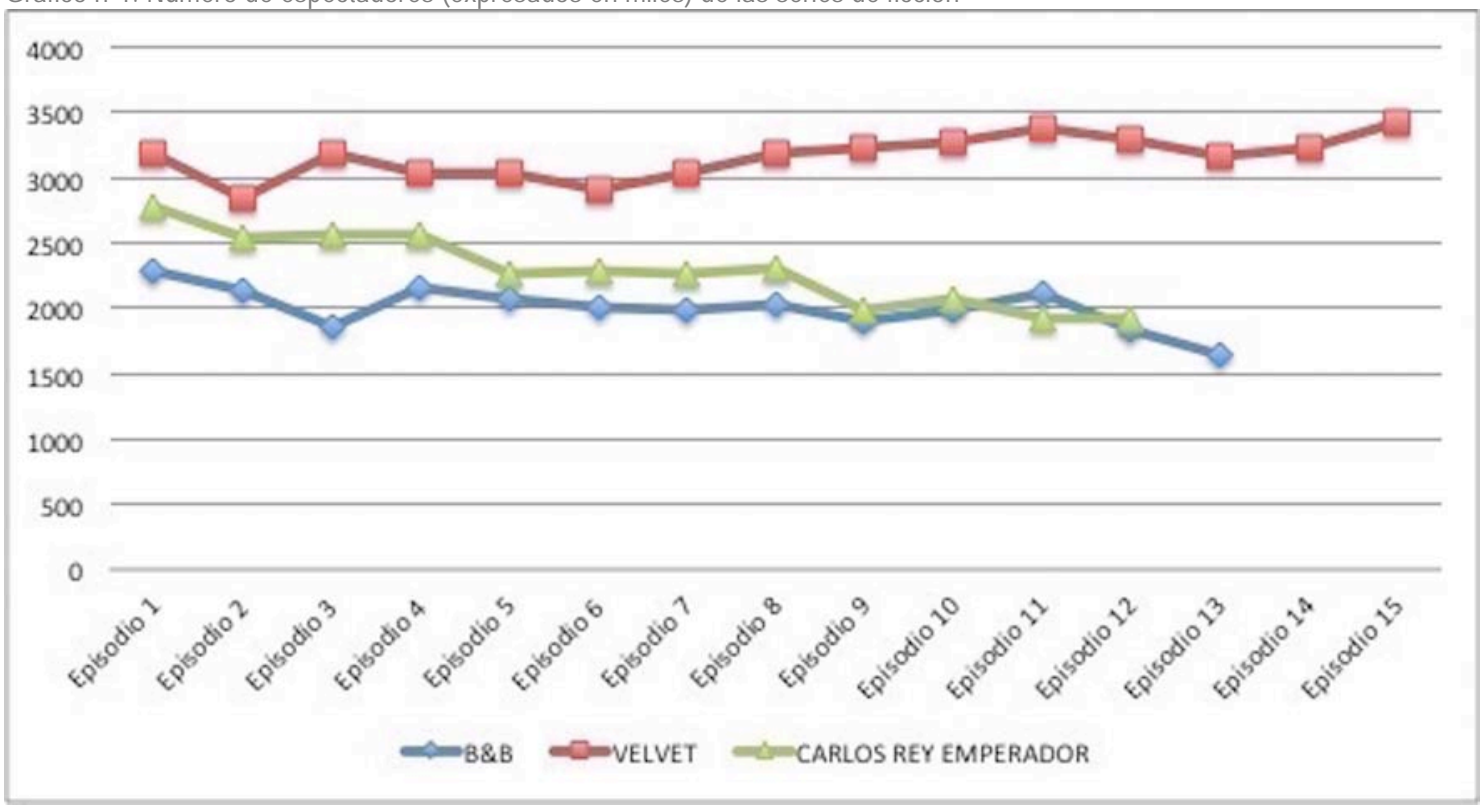

Fuente: Kantar Media. Elaboración propia.

Por lo que respecta a la audiencia lineal, excepto en Velvet, en el resto se aprecia un desgaste notable de las series a medida que avanzan los capítulos ya que en general experimentan una tendencia a la baja, sobre todo en $B \& B$. Los continuos cambios en el horario de programación de la serie en la parrilla de Telecinco dificultaron el seguimiento de la misma por parte del espectador. Los datos de $B \& B$, la serie con peores datos de las tres analizadas, indican que su primer episodio es el que alcanza un mejor registro (2.283.000 espectadores), mientras que el más bajo es el último (1.637.000 espectadores), cifras muy inferiores a las conseguidas por cualquier emisión en prime time de Telecinco. Por otra parte, Carlos Rey Emperador durante el periodo analizado también obtiene su mejor dato el día de su estreno (2.783.000 espectadores) y una cuota de pantalla de 15,6\% pero pierde paulatinamente televidentes hasta llegar a su registro mínimo de 1.917.000 espectadores el 16 de noviembre de 2015. Esta ficción pretendía emular el éxito alcanzado por la serie histórica Isabel que supuso a la cadena pública reunir a un público fiel en años anteriores.

A diferencia de las anteriores, la serie de Antena 3 Velvet no obtiene los mejores datos el día de su estreno sino durante la emisión del último episodio (3.427.000 espectadores) y el peor tan solo una semana después de su estreno (17-09-2015) con un 16,1\% de cuota de pantalla y 2.839.000 espectadores, probablemente debido a la retransmisión por parte de Cuatro del partido de Eurobasket España-Francia 
Gráfico n². Audiencia social única total (expresada en miles) de las series de ficción

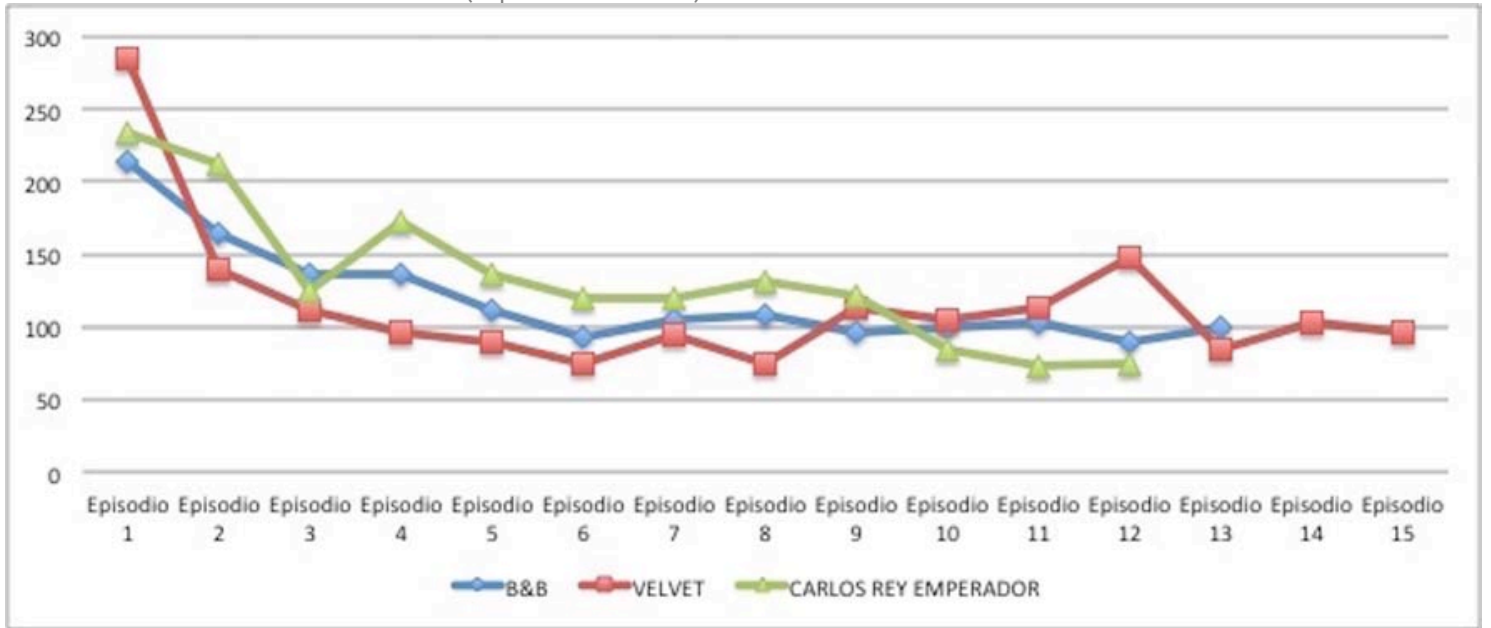

Fuente: Kantar Media. Elaboración propia.

Con respecto a la audiencia social, centrada en Twitter, en los tres casos se percibe una caída a partir del primer episodio, descenso muy notable en el caso de Velvet que reduce a la mitad su audiencia única social. La razón del éxito de ese primer episodio pudiera ser que Telecinco todavía no había comenzado con uno de sus formatos estrella, Gran Hermano, cuyo estreno coincide con la emisión del segundo episodio de la serie de Antena 3. Este programa siempre ha alcanzado una de las cuotas más altas de audiencia social en la programación generalista española de los últimos años.

Por el contrario, la subida que experimenta en el episodio 12 probablemente se deba a un importante giro en la trama por la desaparición de la serie de uno de sus protagonistas, demostrando así que la audiencia parece ser más sensible a las tramas sentimentales.

Junto a los resultados reflejados en el gráfico cabe destacar también los indicadores recogidos en la siguiente tabla que ayudan a valorar globalmente el comportamiento de la audiencia social:

Tabla n¹. Indicadores de la audiencia social de las tres series de ficción (octubre-diciembre 2015)

\begin{tabular}{|c|c|c|c|c|c|c|c|c|c|}
\hline & $\begin{array}{l}\text { Twoests } \\
\text { Totales }\end{array}$ & $\begin{array}{l}\text { Impreslon. } \\
\text { Totales }\end{array}$ & $\begin{array}{l}\text { Imprealón. } \\
\text { Totales } \\
\text { (modla) }\end{array}$ & $\begin{array}{l}\text { Audiencia } \\
\text { Ôniea } \\
\text { Total }\end{array}$ & Aut & $\begin{array}{l}\text { Autores } \\
\text { Ûnicos } \\
\text { por } 1000 \\
\text { espect. }\end{array}$ & $\begin{array}{l}\text { Sont. } \\
\text { positivo }\end{array}$ & $\begin{array}{l}\text { Sont } \\
\text { negatlvo }\end{array}$ & $\begin{array}{l}\text { Sent } \\
\text { neutro }\end{array}$ \\
\hline$B 8 B$ & 49.660 & 5.044 .930 & 388.072 & 80.068 & 1.680 & 0,9 & $28,6 \%$ & $6,5 \%$ & $65 \%$ \\
\hline Velvet & 41.698 & 6.926 .763 & 577.230 & 99.046 & 1.622 & 0,5 & $22,7 \%$ & $8,4 \%$ & $68,9 \%$ \\
\hline Carlos & 45.534 & 6.211 .941 & 690.216 & 101.719 & 1.430 & 0,7 & $19,7 \%$ & $5,8 \%$ & $74,5 \%$ \\
\hline
\end{tabular}

Fuente: Kantar Media. Elaboración propia

Frente a los excelentes resultados de audiencia lineal, Velvet no alcanza el primer lugar en la mayor parte de los datos procedentes de la audiencia social. La diferencia existente en las otras audiencias entre esta serie y las demás desaparece en el caso de la audiencia social. Son los espectadores de $B \& B$ los más activos en Twitter y los que producen el mayor número de tweets de las tres series analizadas (49.690). Sin embargo, Velvet es la ficción con mayor número de impresiones totales (6.926.763).

Es la serie histórica de Carlos Rey Emperador la que menos reacciones, tanto positivas como negativas, provoca entre la audiencia pero es la ficción que cuenta con una audiencia única superior (101.719). 


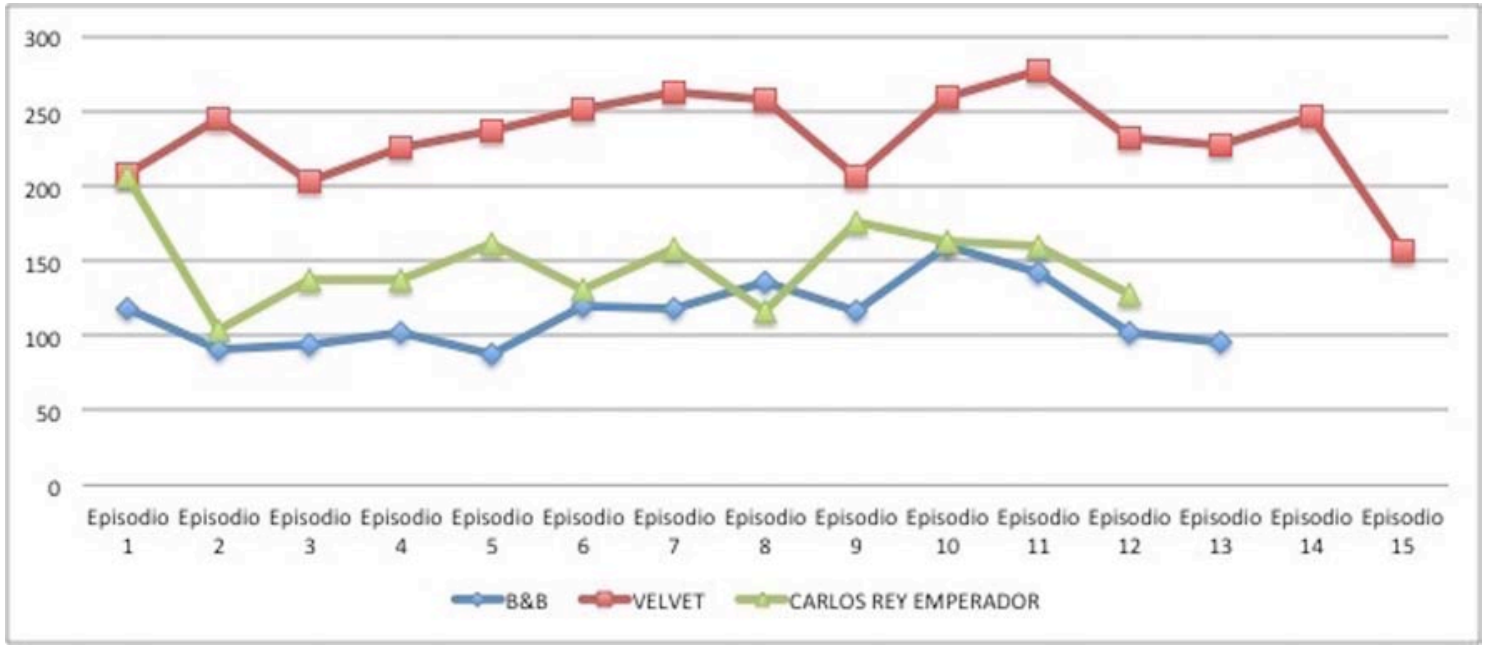

Fuente: Kantar Media. Elaboración propia.

En relación a la audiencia en diferido, al igual que había sucedido con la audiencia lineal, Velvet obtiene los mejores datos, y B\&B los peores en la mayor parte de los episodios. En general, la audiencia en diferido de esta ficción de Atresmedia supone el 7-8\% de la audiencia lineal. Este mismo porcentaje se sitúa entre el 4,75\% y el 6,69\% en el caso de B\&B. Mientras que Carlos Rey Emperador obtiene en este tipo de datos entre el 5 y $8 \%$ de su audiencia lineal.

Un dato resaltable corresponde al último episodio de cada una de las series, uno de los más bajos durante este periodo. Se puede suponer que los espectadores prefieren ver los finales de temporada en directo, donde habitualmente se resuelve la trama principal de la serie, sin tener que esperar a su visionado en diferido.

Gráfico n²4. Número de espectadores (expresados en miles) de la audiencia lineal (A.L.) y audiencia en diferido (A.D.) de las series de ficción

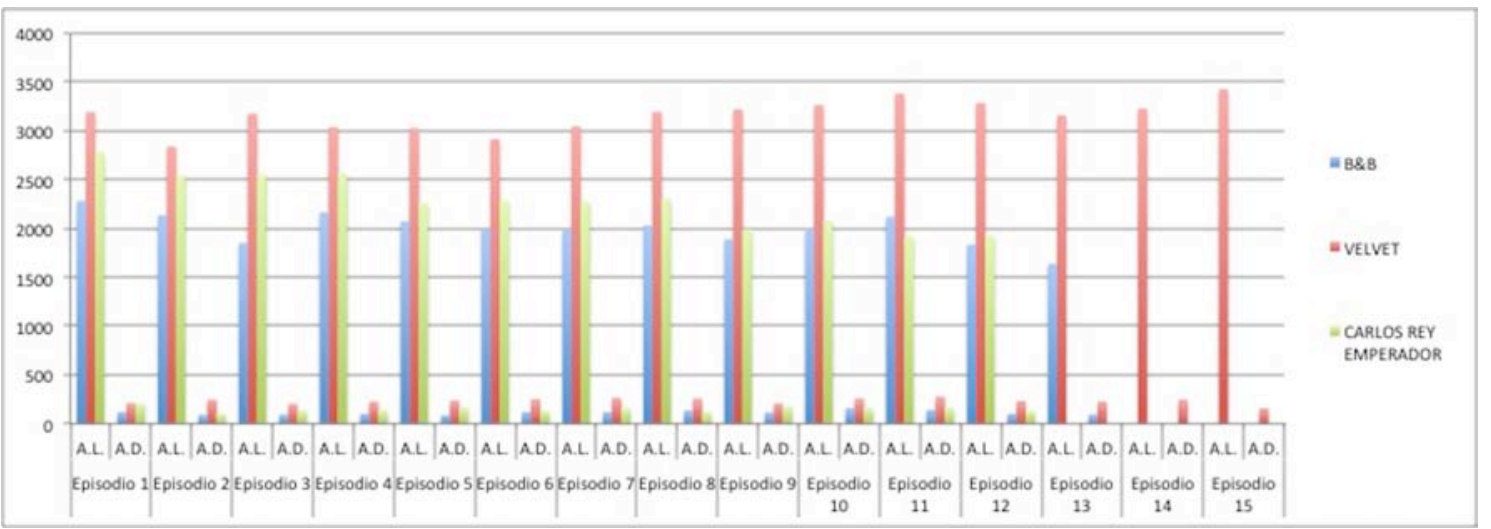

Fuente: Kantar Media. Elaboración propia.

Según los datos obtenidos sobre el comportamiento de la audiencia lineal y en diferido se puede concluir que ambas se complementan. Por ejemplo, el episodio 2 de Velvet baja en audiencia lineal pero sube en diferido y su último episodio tiene el dato más bajo de audiencia en diferido y el más alto en lineal. En el caso de Carlos Rey Emperador, el episodio 9 es uno de los más bajos en la audiencia lineal mientras que fue de los más vistos en los días posteriores a su emisión. En el caso de $B \& B$ no se aprecia dicha complementación de las audiencias. Es curioso que Velvet no mantenga esa posición líder en la audiencia social. Es más, durante siete semanas es la ficción analizada con peores datos en este tipo de audiencia. Es probable que la competencia de Gran Hermano, formato estrella que triunfa siempre en las redes sociales, frene sus datos. La competencia de este reality impulsa a algunos de los seguidores de la serie a consumirla en diferido, dejando el directo de Gran Hermano para el consumo lineal. En este sentido, cabe recordar que Telecinco ha sido la cadena más comentada del año 2015 (con más de 30 millones de tuits) gracias a sus realities ya que uno de cada cuatro tuits sobre televisión social está relacionado con este formato (Kantar Media, 2016).

Si comparamos los datos obtenidos de la audiencia en diferido con los de otros países europeos, comprobamos que el consumo es muy similar. En un estudio realizado en 26 países, este tipo de audiencia incrementaba un 7\% la audiencia tradicional, porcentaje que también muestran las series analizadas (Eurodatatv, 2016). 


\section{Conclusiones}

Al margen de los cambios continuos en la configuración de las parrillas televisivas llevadas a cabo por las cadenas de televisión para lograr concentrar al mayor número de espectadores que influyen directamente en los resultados de las series de ficción analizadas, de la investigación realizada se concluye que si bien la audiencia lineal sigue siendo la principal, los datos procedentes de la social y el consumo en diferido completan y complementan los datos de la primera. No obstante, en ningún caso la audiencia social o en diferido produce vuelcos significativos en las clasificaciones de las ficciones más vistas en general. Diversos estudios han comprobado que la audiencia social tiene una influencia directa en los índices de audiencia lineal y diferido (Neira, 2015). Por lo tanto, estas nuevas audiencias típicas de la televisión líquida provocan unos flujos que confluyen en la audiencia matriz lineal.

En estos momentos de cambios en el panorama televisivo quedan muchos retos por abordar. Si bien es cierto que la publicación de los datos de audiencia en diferido nos acercan cada vez más a la realidad de las audiencias actuales, cabe precisar que en España solo el $65 \%$ de los hogares tienen capacidad para este visionado (Zenith, 2015). El panorama de las audiencias tendría que completarse además con los datos sobre el consumo ampliado a través de otros dispositivos móviles como tabletas, smartphones o portátiles. También sería conveniente extender el periodo de recolección de datos tanto de la audiencia en diferido a más de 7 días, como de la audiencia social (treinta minutos antes y treinta minutos después del horario de emisión), de modo que los datos se acercaran aún más al comportamiento de los usuarios.

Nos encontramos en un momento de transición en el que los cambios se suceden vertiginosamente. Las alianzas como la recientemente establecida entre Comscore y Kantar Media para la medición crossmedia van en esta dirección para conseguir extraer más información dentro de la corriente Big Data. Otro de los retos del futuro sería la inclusión de los nuevos actores de la televisión líquida como los operadores OTT (Netflix), Youtube, Apple tv o Nexus Player.

Cabe indicar que las estrategias de programación de las diferentes cadenas influyen en los resultados obtenidos ya que chocan con las metodologías de medición, por ejemplo en el caso de la audiencia social. El impacto en redes sociales de un modelo de programación más circular como el de Mediaset, en la que las emisiones como los reality son objeto de debate y análisis en otros programas, más allá del momento de emisión, no viene recogido en las estadísticas de la audiencia social, por lo que una parte de esa audiencia generada en las redes sociales no se contempla en los informes.

Asimismo, el estudio de estas audiencias puede influir en la modificación del mercado televisivo de modo que haya formatos más proclives al directo y otros más al diferido. Mientras en la audiencia social suelen tener éxito los acontecimientos en directo (Calatrava \& Chaves, 2016), los resultados relativos al consumo en diferido indican todo lo contrario (a excepción del estreno de alguna serie).

En definitiva, este artículo supone una de las primeras investigaciones sobre el análisis del comportamiento de estas tres tipologías de audiencias en el ámbito español. Aunque esta investigación está ligada a un producto audiovisual determinado, las series de ficción, permite aproximarnos al conocimiento y comportamiento de los tres tipos de audiencias. Sin embargo, sería de gran interés ampliar esta investigación a otros géneros y formatos televisivos para tratar de establecer patrones de comportamiento de estas nuevas audiencias a la vez que se determina cuáles pueden ser las repercusiones que tendrá el comportamiento de estas audiencias en el mercado publicitario con el fin de valorar cuál es el modelo de negocio que se va a implantar en este nuevo entorno digital. Asimismo, sería interesante contar con más información de corte cualitativo sobre el perfil de las personas que realizan el visionado en diferido o que comentan en Twitter un espacio televisivo.

Para finalizar, con el fin de abrir nuevas líneas de investigación y dado que los jóvenes son uno de los segmentos de la población que más apuestan tanto por la audiencia social como por el consumo en diferido, se hace necesario apostar por las investigaciones que expliquen su comportamiento ya que serán los usuarios del mañana. En un contexto de descenso de visionado tradicional, se debe buscar a dónde se trasladan las audiencias y realizar estudios que nos aproximen a su comportamiento.

\section{Bibliografía}

Abreu, J., Nogueira, J., Becker, V., \& Cardoso, B. (2016). Survey of Catch-up TV and other time-shift services: a comprehensive analysis and taxonomy of linear and nonlinear television. Telecommunication Systems, 1-18.

Andò, R. (2014). What does TV actually mean? New consumer experience and generations. Participations, 11(2), 156-181.

Alonso González, M. (2014). Audiencia Social: el telespectador comienza a participar en los contenidos televisivos. Ambitos: Revista internacional de comunicación, 25, 77.

Barra, L., \& Scaglioni, M. (2014). TV Goes Social: Italian Broadcasting Strategies and the Challenges of Convergence. VIEW Journal of European Television History and Culture, 3(6), 110-124.

Bredl, K., Ketzer, C., Hünniger, J., \& Fleischer, J. (2014). Twitter and Social TV. Microblogging as a new approach to audience research. En G. Patriarche, H. Bilandzic, J. Linaa Jensen, \& J. Jurisic (eds.), Audience Research Methodologies. Between innovation and consolidation (pp. 196-211). New York: Routledge.

Calatrava, A. \& Chaves, L. (2016). ¿Sabías que la inversión en Twitter, combinada con publicidad en TV, genera más ventas para las marcas?. 32 seminario de televisión multipantalla. Girona

Carey, J. (2016). Audience measurement of digital TV. International Journal of Digital Television, 7(1), 119-132.

Claes, F., \& Deltell, L. (2015). Audiencia social en Twitter: hacia un nuevo modelo de consumo televisivo. Trípodos, 36, 109-132.

Coller, X. (2000). Estudio de casos. Madrid: Centro de Investigaciones Sociológicas.

Díaz Nosty, B. (2015). Tendencias de futuro. Medios continuos y nuevo paradigma. Telos: Cuadernos de comunicación e innovación, (100), 58-61.

EurodataTV (2016). One TV Year in the world : a wealth of television content expands its journey to new screens around the world. 
Recuperado de: http://www.mediametrie.com/eurodatatv/communiques/one-tv-year-in-the-world-a-wealth-of-television-content-expands-itsjourney-to-new-screens-around-the-world.php?id=1440

Deltell, L., Claes, F., \& Congosto, M. L. (2015). Enjambre y urdimbre en twitter: análisis de la audiencia social de los premios Goya 2015. En Quintas-Froufe, N. \& González-Neira, A. (coord.). La participación de la audiencia en la televisión: de la audiencia activa a la social (pp. 60-82). Madrid: AIMC.

Evens, T., \& Berte, K. (2014). Challenges of Digital Innovations: a Set-Top Box Ba-sed Approach. En: J. Bourdon, and C. Meadel (eds.). Television Audiences across the World: De-constructing the Ratings Machine (pp. 234-247). Basingstoke: Palgrave Macmillan.

Gallardo-Camacho, J., Lavín, E., \& Fernández-García, P. (2016a). Los programas de televisión deportivos y su relación con la audiencia social en Twitter en España. Revista Latina de Comunicación Social, 71, 272-286. doi: 10.4185/RLCS-2016-1095

Gallardo-Camacho, J., Lavín, E., \& Fernández-García, P. (2016b). Análisis de los programas de televisión más comentados en Twitter y su relación con la audiencia tradicional en España. Palabra Clave, 19(1), 185-210. doi: 10.5294/pacla.2016.19.1.8

Gómez Rubio, L., \& López Vidales, N. (2015) Del éxito en televisión a la participación en las redes sociales. El Príncipe y Galerías Velvet en Facebook. Doxa Comunicación, 20, 136-160.

González-Neira, A. \& Quintas-Froufe, N. (2014). Audiencia tradicional frente a audiencia social: un análisis comparativo en el prime-time televisivo. Mediterranean Journal of Communication, 5 (1), 105-121. doi:10.14198/MEDCOM2014.5.1.02

González-Neira, A. \& Quintas-Froufe, N. (2015). La audiencia social en España e Italia: análisis comparativo. En Nuevos perfiles y audiencias para una democracia participativa. VII Actas Congreso Internacional Ciberperiodismo y web 2.0. (pp. 143-155). Bilbao: Universidad del País Vasco.

Iñesta Fernández, N. (2015). Espectador social y convergencia televisiva. Ambitos: Revista internacional de comunicación, 28, 50-62.

Kantar Media (2016). Anuario Social TV (2015). Recuperado de: http://www.kantarmedia.com/es/blog-y-recursos/data-lab/anuario-social-tv Kosterich, A. (2016). Reconfiguring the "Hits": the new portrait of television program success in an era of big data. International Journal on Media Management, 1-16. doi:10.1080/14241277.2016.1166431

Kosterich, A., \& Napoli, P. M. (2016). Reconfiguring the audience commodity: The institutionalization of social TV analytics as market information regime. Television \& New Media, 17(3), 254-271. doi:10.1177/1527476415597480

López-Meri, A. (2015). El impacto de Twitter en el periodismo: un estado de la cuestión. Revista de la Asociación Española de Investigación de la Comunicación, (2)4, 34-41.

Madinaveitia, E., \& Merchante, M. (2015). Medición de audiencias: desafío y complejidad en el entorno digital. Harvard Deusto Marketing y Ventas, 131, 26-33.

Marinelli, A., \& Andò, R. (2016). La nuova centralità televisiva. Schermi, contenuti, practiche delle audience connesse. Roma: Osservatorio SocialTV. Università della Sapienza.

Min, J., Zang, Q., \& Liu, Y. (2015, April). The influence of social media engagement on TV program ratings. In Systems and Information Engineering Design Symposium (SIEDS), 2015 (pp. 283-288). IEEE.

Monzoncillo, J. M. (2011). Las nuevas televisiones: personalización e individualización. En J.M. Monzoncillo (coord.), La televisión etiquetada: nuevas audiencias, nuevos negocios (pp. 93-101). Barcelona: Ariel.

Neira, E. (2015). La otra pantalla. Redes sociales, móviles y la nueva televisión. Barcelona: UOC.

Portilla, I. (2015). Television Audience Measurement: Proposals of the Industry in the Era of Digitalization. Trípodos, 36, 75-92.

Quintas-Froufe, N. \& González-Neira, A. (coord.) (2015). La participación de la audiencia en la televisión: de la audiencia activa a la social. Madrid: AIMC.

Saavedra, M., Rodríguez, L., \& Barón, G. (2015). Audiencia social en España: Estrategias de éxito en la televisión nacional. Revista ICONO14. Revista científica de Comunicación y Tecnologías emergentes, 13 (2), 214-237. doi: 10.7195/ ri14.v13i2.822

Simons, N. (2015). TV drama as a social experience: An empirical investigation of the social dimensions of watching TV drama in the age of non-linear television. Communications, 40(2), 219-236. doi: 10.1515/commun-2015-0005

Steele, L., James, R., Burrows, R., Mantell, D. L., \& Bromham, J. (2015). The consumption of on-demand. Journal of Promotional Communications, 3(1), 219-241.

Suing, A., Gallardo, D., \& Ortiz, C. (2016). La televisión social en Ecuador, análisis de participación en programas de entretenimiento. Revista ComHumanitas, 6(1), 149-169.

Woodford, D., Goldsmith, B., \& Bruns, A. (2015). Social Media Audience Metrics as a New Form of TV Audience Measurement. Produsing Theory in a Digital World 2.0: The Intersection of Audiences and Production in Contemporary Theory, 99, 141-158.

Yin, R. (1989). Case studies research. Newbury Park, CA: Sage Publications.

Zenith (2016). La televisión que vivimos. Recuperado de: http://www.slideshare.net/ZenithES/la-televisin-que-vivimos-2015

Agradecimientos

Agradecemos a Kantar Media la cesión de los datos para la realización de esta investigación.

\section{Cómo citar este artículo en bibliografías - How to cite this article in bibliographies / references:}

GONZÁLEZ-NEIRA, A.; QUINTAS-FROUFE, N. (2016): "El comportamiento de la audiencia lineal, social y en diferido de las series de ficción españolas". En Revista de la Asociación Española de Investigación de la Comunicación, vol. 3, número 6, pp. 27-33. 